\title{
Anthocyanin Accumulation and Transcriptional Regulation of Anthocyanin Biosynthesis in Purple Pepper
}

Boyan Tang ${ }^{1,2}$, Ling $\mathrm{Li}^{1,2}$, Zongli Hu${ }^{1,2}$, Yanan Chen ${ }^{1,2}$, Tingting Tan ${ }^{1,2}$, Yanhua Jia ${ }^{1,2}$, Qiaoli Xie ${ }^{1,2^{*}}$ and Guoping Chen ${ }^{1,2^{*}}$

${ }^{1}$ Key Laboratory of Bioengineering Science and Technology, Chongqing University, Ministry of Education

${ }^{2}$ Bioengineering College, Campus B, Chongqing University, 83 Shabei Street, Chongqing 400045, People's Republic of China.

*Corresponding author. Guoping Chen, Tel:00862365112674; Fax:0086 23 65112674;

E-mail: chenguoping@cqu.edu.cn. Qiaoli Xie, E-mail: qiaolixie@cqu.edu.cn. 
Supplementary Table 1. Primers used for qRT-PCR analysis of anthocyanin biosynthesis-related genes in pepper.

\begin{tabular}{|c|c|c|}
\hline Genes & $\begin{array}{l}\text { Forward primer ( } 5^{\prime} \text { to } 3^{\prime} \text {, top), } \\
\text { reverse primer ( } 5^{\prime} \text { to } 3^{\prime} \text {, bottom) }\end{array}$ & Accession \\
\hline \multirow[t]{2}{*}{ CaPAL } & CAGATTGAGGCTGCTGCTATTA & LOC107843092 \\
\hline & GGAGATGTTCGGAGAGCATAAC & \\
\hline \multirow[t]{2}{*}{$\mathrm{CaC} 4 \mathrm{H}$} & TATCCTAGCGCTGCCAATTC & LOC107875407 \\
\hline & GACTGAACTGTCCACCTTTCTC & \\
\hline \multirow[t]{2}{*}{ Ca4CL } & GTGGATCCGGATACGGGTTG & LOC 107862076 \\
\hline & AACCCCATATCGCCAGTGTG & \\
\hline \multirow[t]{2}{*}{$\mathrm{CaCHS}$} & GTTGCTTTGCTGGTGGAACC & LOC 107871256 \\
\hline & GTATCACTTGGGCCACGGAA & \\
\hline \multirow[t]{2}{*}{$\mathrm{CaCHI}$} & TTCCTTGCTGGGATTAGAGGG & LOC 107852750 \\
\hline & CCCGTCAAGGGCGTAATCAT & \\
\hline \multirow[t]{2}{*}{$\mathrm{CaF3H}$} & ACCCTTGGGTTGAAAAGGCA & LOC 107859880 \\
\hline & TCTTGAACCTCCCGTTGCTC & \\
\hline \multirow[t]{2}{*}{$\mathrm{CaF3} \mathrm{H}$} & CTACCCCCTGGTCCAAAACC & LOC107862334 \\
\hline & TAAGAACTGAGCCGCCACTG & \\
\hline \multirow[t]{2}{*}{$\mathrm{CaF3} 5 \mathrm{YH}$} & TTAATAGCCATCGGACAGGGG & LOC107848667 \\
\hline & AGCCATACCACAAGTGCCAA & \\
\hline \multirow[t]{2}{*}{ CaDFR } & CGGCTGGATTTATCGGCTCT & LOC107860031 \\
\hline & CTTCCACGGTCAAGTCTGCT & \\
\hline \multirow[t]{2}{*}{ CaANS } & TCCCCCATCGTTTCGTGAAG & LOC 107843451 \\
\hline & AATTGTTTCCTGACGCACGC & \\
\hline \multirow[t]{2}{*}{ CaUFGT } & TTTGGTAGCCATGCAGTTCC & LOC107843659 \\
\hline & TAGGGGTGTCATTGCCTTGT & \\
\hline \multirow[t]{2}{*}{ CaANT1 } & CGTTGCAAGTAAGGAAAGGTGC & LOC 107854818 \\
\hline & CTAGCAGGAACAAGGTGCCA & \\
\hline $\mathrm{CaANT2}$ & TTGTGAAAAGCCAACAGCCG & LOC107844901 \\
\hline
\end{tabular}


CCCAACCATCACTCTGTCCC

CaAN1

ACCTCCAATCCCACCACCTTT

LOC 107842687

TTCTCCCGCAATTCTACCATC

CaTTG1

AGGCATCAAGCGAGTGTGAA

LOC107862994

CAAATAAGCGCCTGCCCATC

Supplementary Table 2. Primers used for construction of yeast expression vectors.

\begin{tabular}{|c|c|c|}
\hline Genes & $\begin{array}{l}\text { Forward primer (5' to } 3^{\prime} \text {, top), } \\
\text { reverse primer (5' to } 3^{\prime} \text {, bottom) }\end{array}$ & Accession \\
\hline \multirow[t]{2}{*}{ CaANT1 } & TCCCCCGGGTGGACTTGTTATGAATACTCCAAT & LOC 107854818 \\
\hline & CGGGATCCGTCCATAAGACAAGCGAGG & \\
\hline \multirow[t]{2}{*}{ CaANT2 } & TCCCCCGGGATGAACAGTACTCCGATTTTGGG & LOC107844901 \\
\hline & CGGGATCCTTTGAACCCCTACAGCAAACC & \\
\hline \multirow[t]{2}{*}{ CaAN1 } & TCCCCCGGGACCATCTGGTAGTCACCAACC & LOC 107842687 \\
\hline & CGGGATCCTCCAAGTCACACAGGCATGA & \\
\hline \multirow[t]{2}{*}{ CaTTG1 } & TCCCCCGGGTCACTATCCCCTAACAAAAAATGG & LOC107862994 \\
\hline & CGGGATCCCTTTACAAGATTGCTCATCTCCAA & \\
\hline
\end{tabular}

Supplementary Table 3. Primers used for construction of BiFC vectors.

\begin{tabular}{|c|c|c|}
\hline Genes & $\begin{array}{l}\text { Forward primer (5' to } 3^{\prime} \text {, top), } \\
\text { reverse primer (5' to } 3^{\prime} \text {, bottom) }\end{array}$ & Accession \\
\hline CaANT1 & $\begin{array}{l}\text { GAGTGAGCTCATGAATACTCCAATAATCTGTACAA } \\
\text { CGCGGATCCATTAAGTAGATTCCATATGTCATCC }\end{array}$ & LOC107854818 \\
\hline CaANT2 & $\begin{array}{l}\text { GAGTGAGCTCATGAACAGTACTCCGATTTTGG } \\
\text { CGCGGATCCATCAAGTAGATCCCATAGGTCAATA }\end{array}$ & LOC107844901 \\
\hline CaAN1 & $\begin{array}{l}\text { GAGTGAGCTCATGATGGAGATCATACAGCCTA } \\
\text { CGCGGATCCGGGATTATCTGATGTATTGACTTT }\end{array}$ & LOC107842687 \\
\hline CaTTG1 & $\begin{array}{l}\text { GAGTGAGCTCATGGAAAATTCAAGCCAAGAA } \\
\text { CGCGGATCCTACTTTAAGCAGCTGCAACTTG }\end{array}$ & LOC107862994 \\
\hline
\end{tabular}


